\title{
Point field models for the galaxy point pattern
}

\section{Modelling the singularity of the two-point correlation function}

\author{
M. Snethlage ${ }^{1}$, V. J. Martínez ${ }^{2}$, D. Stoyan ${ }^{3}$, and E. Saar ${ }^{4}$ \\ 1 Mathematical Department, Silesian University at Opava, Bezručovo nám. 13, 74601 Opava 1, Czech Republik \\ e-mail: Martin.Snethlage@math.slu.cz \\ 2 Observatori Astrònomic, Universitat de València, 46100 Burjassot, Spain \\ 3 Institut für Stochastik, TU Bergakademie Freiberg, Bernhard-von-Cotta-Str. 2, 09596 Freiberg, Germany \\ e-mail: stoyan@orion.hrz.tu-freiberg.de \\ 4 Tartu Observatory, Tõravere, 61602, Estonia \\ e-mail: saar@aai.ee
}

Received 7 December 2001 / Accepted 8 April 2002

\begin{abstract}
There is empirical evidence that the two-point correlation function of the galaxy distribution follows, for small scales, reasonably well a power-law expression $\xi(r) \propto r^{-\gamma}$ with $\gamma$ between 1.5 and 1.9. Nevertheless, most of the point field models suggested in the literature do not have this property. This paper presents a new class of models, which is produced by modifying point fields commonly used in cosmology to mimic the galaxy distribution, but where $\gamma=2$ is too large. The points are independently and randomly shifted, leading to the desired reduction of the value of $\gamma$.
\end{abstract}

Key words. galaxies: statistics - large-scale structure of universe - methods: statistical

\section{Introduction}

The two-point correlation function $\xi(r)$ has been the primary tool for quantifying large-scale cosmic structure (see Peebles 1980). Several estimates of this statistical quantity from redshift surveys suggest a power-law shape of $\xi(r)$ for $0.5 h^{-1} \mathrm{Mpc}<r<10 h^{-1} \mathrm{Mpc}$ ( $h$ being the Hubble constant in units of $100 \mathrm{~km} \mathrm{~s}^{-1} \mathrm{Mpc}^{-1}$ )

$\xi(r)=\left(\frac{r}{r_{0}}\right)^{-\gamma}$

with $\gamma$ between 1.5 and 1.9, see for example Davis \& Peebles (1983), Maddox et al. (1990), Martínez et al. (1993) or Pons-Bordería et al. (1999). Thus, $\xi(r)$ seems to have a singularity of order $\gamma$. However, taking into account that galaxies have a physically finite extended size, the shape of $\xi(r)$ has necessarily to deviate, at very small scales, from the power-law behavior.

It is important to be able to generate controllable point processes resembling the galaxy distribution, not only showing visual similar patterns to the observed one, but also reproducing the same statistical properties. These

Send offprint requests to: V. J. Martínez,

e-mail: Vicent.Martinez@uv.es processes are necessary to test the performance and, in particular, the discriminative power of the statistical estimators used to describe the cosmic texture (Martínez \& Saar 2002). It is also worth mentioning that two point fields could present similar second-order characteristics, such as the two-point correlation function analyzed in this paper, but show completely different results for higher order statistics.

Several methods have been published so far to generate mock catalogues to mimic the galaxy distribution. We could distinguish two main kinds of methods: Those based on a physically motivated procedure, and those based on a stochastic model with controllable statistical properties. The first family of methods includes the following two main approaches:

1. Starting with cosmological $N$-body simulations based on the gas-dark matter dynamics, one can extract galaxy catalogues by applying physical schemes related with the galaxy formation processes (Kauffmann et al. 1999). This is obviously the most realistic way to obtain mock representations of the galaxy distribution. However, it requires much computing time and therefore the cost of obtaining several realizations is high.

2. The second possibility is to treat the dynamics of the non-linear clustering of matter by means of 
approximate methods based on higher order perturbation theory. A recent example is the so-called PThalo model introduced by Scoccimarro \& Sheth (2002). These authors select virialized dark matter halos from simulations obtained by applying the second-order Lagrangian perturbation theory. Then, given a biasing scheme which accounts for the fact that the galaxy formation needs not to be proportional to the local matter density, one can extract realistic galaxy distributions.

The second family of models for the galaxy distribution are those which are based on statistical or geometrical prescriptions. These models are more flexible than those from the first family, can be easily simulated and manipulated, and have more controllable statistical properties. In many cases, these statistics can be calculated analytically. The first model of this family was introduced by Neyman \& Scott (1958) and became very popular in spatial statistics. The characteristics of this model were probably prefigured by the Lick survey. Peebles (1980) reviews two point processes providing clustering patterns with power-law behavior for the two-point correlation function: the Soneira and Peebles hierarchical model (Soneira \& Peebles 1978) and the Mandelbrot's prescription based on the RayleighLévy random walk (Mandelbrot 1982). The first model, based on a superposition of several fractal clumps, reproduces well the second-, third-, and fourth-order correlation functions. In the Rayleigh-Lévy model, galaxies are placed at the steps of a random walk starting from an initial position, where each jump takes an isotropically chosen random direction with the length following a power-law probability distribution function. Although this model shows point distributions with little resemblance to the observed one, it has been successfully applied to test the error measurements of counts-in-cells statistics (Szapudi \& Colombi 1996). The singularity of the correlation function in these models can be varied in a rather wide interval.

Other point field models published in literature present a two-point correlation function without any singularity or with a singularity of order 2 . A well-known example is the Cox segment process, where points are situated on randomly oriented line segments. Its correlation function was derived by Stoyan et al. (1995) and has a singularity of order 2. A similar cluster process was introduced by Buryak \& Doroshkevich (1996), where the points (i.e., the galaxies) populate a mixture of randomly oriented lines and planes. The singularity of the correlation function is determined by the line component and has again the order 2 .

Van de Weygaert (1991) suggests a point field model which is built from the vertices of a Voronoi tessellation calculated from a randomly distributed nuclei. This model, based on one of the most interesting constructions studied in stochastic geometry, has the property of mimicking the statistical properties of the distribution of clusters of galaxies. However, for this model, the order of the singularity of $\xi(r)$ is again 2 .

A still simpler point field model is appearing in Stoyan (1992). It is a Poisson cluster process where each cluster consists of only two points. The corresponding two-point correlation function can be given a singularity of any desired order between 0 and 3. Of course, this point field is rather primitive and has no physical motivation. It makes sense only with regard to the singularity of the two-point correlation function.

This paper investigates an idea which leads to a new class of point field models. They can have any desired value of $\gamma$ between 0 and 3 and seem to be more realistic than the previous models.

The models listed above are based on simplifying assumptions which are not well fulfilled in reality. The idea is now to model the difference between ideal and real world by means of an additional random element, i.e., by giving independently a random shift to each point of the theoretical model.

The analytical investigation of the effect of these random shifts is very difficult and is therefore carried out only for the case of relatively simple Poisson cluster processes, the Neyman-Scott processes (see Martínez \& Saar 2002 for a description of these processes). It will be shown that a suitable shift of the points will reduce $\gamma$. However, the shift may also be too big and completely destroy the singularity of $\xi(r)$. This is the case, for example, if the shift is Gaussian distributed.

In case of the Voronoi vertices process only simulation based investigations are possible. A segment Cox process is also studied by this means. The analysis performed on both point processes suggest that the effect on $\gamma$ is similar to that for Neyman-Scott processes.

\section{Point field models}

This section presents some point field models whose pair correlation functions have a singularity at $r=0$. All of them have considerably deficiencies with regard either to the exponent in the power-law (see Eq. (1)) or to the underlying physics.

Buryak \& Doroshkevich (1996) suggested a cluster process, where the galaxies are situated on randomly oriented lines and planes of infinite extent. It models the observed fact that galaxies often seem to be situated on filamentary and planar structures (Bhavsar \& Ling 1988). The cluster points form one-dimensional Poisson processes on the lines and two-dimensional Poisson processes on the planes. The two-point correlation function of this point field is proportional to $r^{-2}$ for small $r$, thus $\gamma=2$.

A similar process has been introduced by PonsBordería et al. (1999) for testing the performance of different estimators of the two-point correlation function. A segment Cox point field was used for this purpose. The process is generated by first placing randomly, with a given density $\lambda_{\mathrm{s}}$, segments of fixed length $\ell$, within the window. Secondly, points are scattered randomly on the segments with a given value of the mean number of points per unit length on a segment, $\lambda_{\ell}$. Stoyan et al. (1995) have 
calculated the two-point correlation function of this model which, for $r \leq l$, reads

$\xi(r)=\frac{1}{2 \pi r^{2} \lambda_{\mathrm{s}} \ell}-\frac{1}{2 \pi r \lambda_{\mathrm{s}} \ell^{2}}$.

Again, a value of $\gamma=2$ describes the point process built by the vertices of a Poisson-Voronoi tessellation, see Icke \& Van de Weygaert (1987) and Van de Weygaert \& Icke (1989). The assumption that $\gamma$ is equal to 2 has been verified by Heinrich et al. (1998).

In all these models $\gamma$ is too large. A rather simple point field model which may have any $\gamma$ between 0 and 3 is presented by Stoyan (1994). The points of a homogeneous Poisson process of intensity $\lambda_{p}$ define the centres of independent clusters. Each cluster consists of two points. The two-point correlation function is

$\xi(r)=\frac{f(r)}{8 \pi \lambda_{p} r^{2}}$

where $f(r)$ is the probability density function of the distance between the two cluster points. This point field is a member of the class of Neyman-Scott processes, see Sect. 3.1. The size of $\gamma$ depends on the asymptotic behaviour of $f(r)$ for $r \rightarrow 0$. If $f(r)$ behaves as $r^{\alpha}, \gamma$ is equal to $2-\alpha$. Therefore, a suitable choice of $f(r)$ leads to any desired order. Unfortunately, this point field is very primitive and has no physical motivation. Thus, it is unsuitable to model the galaxy point pattern and is only of technical value. An interesting generalization of this model - the socalled Gauss-Poisson process with clusters of size 0, 1, and 2 - has been presented and analyzed by Kerscher (2001). Kerscher et al. (2001) have shown that this model cannot describe well the distribution of the galaxy clusters.

All of the point fields investigated until now have considerable deficiencies. Those which have the right $\gamma$ are quite unrealistic models for the galaxy point pattern. And those which are appropriate in some astronomical sense have the wrong $\gamma$. The next section provides a method which yields physically motivated models with a two-point correlation function which behaves as desired.

\section{The effect of independent random shifts}

\subsection{Arbitrary shifts applied to Neyman-Scott processes}

This subsection deals with a class of relatively simple Poisson cluster processes, the so-called Neyman-Scott processes (Neyman \& Scott 1958). They result from homogeneous clustering applied to a Poisson process. The points of a homogeneous Poisson process of intensity $\lambda_{\mathrm{p}}$, called parent points, define the centres of clusters. The clusters are independently and identically distributed. A cluster consists of a random number of independently and identically distributed daughter points. The random distance between a daughter point and its corresponding parent point is distributed according to a probability density function $d(r)$. The two-point correlation function of

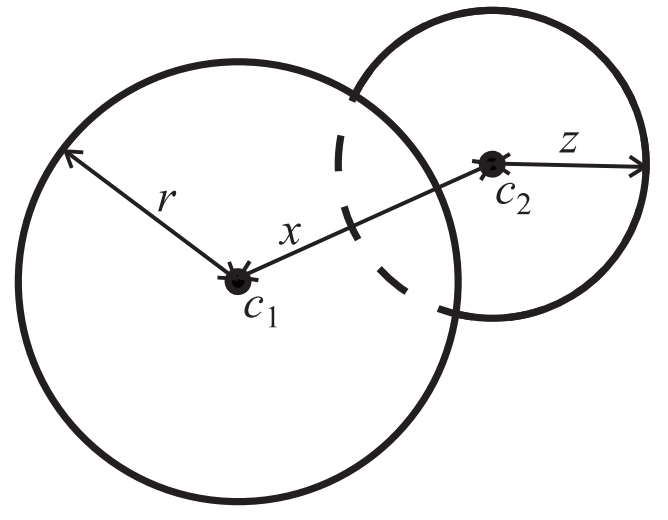

Fig. 1. The quantity $F^{*}(r)$, demonstrated for the twodimensional case. The initial distance between the two cluster points $c_{1}$ and $c_{2}$ is $x$, the length of the shift is $z$. The distance between the shifted and unshifted points is smaller than or equal to $r$ when the shifted point belongs to the dashed line.

such a process is proportional to $f(r) / r^{2}$, see, for example, Stoyan et al. (1995). Here, $f(r)$ is the probability density function of the distance between two randomly selected daughter points of the same cluster.

Given such a Neyman-Scott process, we now apply independent isotropic random shifts to all its points. The isotropic random shift consists of shifting a point by a random length in a uniformly distributed direction. The random length of the shift has a probability density function $d^{*}(r)$. For example, in case of Gaussian shifts $d^{*}(r)$ is the Weibull density function with $p=2$ and $b=1 /\left(2 \sigma^{2}\right)$

$\frac{r^{2}}{\sigma^{2}} \exp \left(-\frac{r^{2}}{2 \sigma^{2}}\right), \quad r \geq 0$

(this is the probability density function of the length of a Gaussian distributed vector in $3-\mathrm{D}$ ).

The random shift changes the distribution of the interpoint distance between two points of the same cluster. We denote the corresponding distribution function by $F^{*}(r)$, and the probability density function by $f^{*}(r)$. The resulting two-point correlation function is proportional to $f^{*}(r) / r^{2}$.

The aim of the following is to determine the asymptotic behaviour of $f^{*}(r)$ for $r \rightarrow 0$. This will yield a recipe how to choose the function $d^{*}(r)$ in order to obtain any desired $\gamma$.

For a first step, assume that every cluster contains exactly two points and that only one of them is shifted. $F^{*}(r)$ is then the probability that the distance between both points is smaller than or equal to $r$. For example, let the initial distance between both points be $x$ and let the shift of length be $z$, see Fig. 1. Then, $F^{*}(r)$ is equal to the fraction of that part of a sphere of radius $z$ centred on the shifted point, which is not farther from the unshifted point than $r$.

As the initial distance between the two cluster points is distributed according to the probability density 
function $f(r)$ and as the length of the shift is distributed by $d^{*}(r)$, we can write

$F^{*}(r)=\int_{-\infty}^{\infty} \int_{-\infty}^{\infty} f(x) d^{*}(z) \frac{A(x ; z ; r)}{4 \pi z^{2}} \mathrm{~d} x \mathrm{~d} z$,

where $A(x ; z ; r)$ is the two-dimensional volume of the intersection of a ball of radius $r$ with a sphere of radius $z$, whose centres are at the distance $x$ :

$$
\begin{aligned}
& A(x ; z ; r)= \\
& \begin{cases}4 \pi z^{2}, & \text { if } x<r-z \text { and } r \geq z \\
\frac{\pi z}{x}\left(r^{2}-(x-z)^{2}\right) & \text { if }|r-z| \leq x \leq r+z \\
0 & \text { otherwise }\end{cases}
\end{aligned}
$$

(the dashed line in Fig. 1).

It is rather complicated to find the integral (5); we describe it in the appendix. The result is that the behaviour of $f^{*}(r)$ for small $r$ depends only on the corresponding behaviours of $d^{*}(r)$ and $f(r)$. If $d^{*}(r)$ behaves as $r^{\alpha}$ and $f(r)$ as $r^{2-\gamma}$ (where $\gamma$ is the order of the singularity of the correlation function before the shifts), then $f^{*}(r)$ behaves as $r^{3-\gamma+\alpha}$, i. e., the order of the singularity of $\xi(r)$ after the shifts is $\gamma-(\alpha+1)$. It has been reduced, as $\alpha+1>0$ (else, $d^{*}(r)$ cannot be a density function).

Now, let the number of daughter points per cluster be arbitrary and let all of them be randomly shifted. As the daughter points are independently distributed, this situation is principally equal to that one before, only with an initial $f(r)$ which behaves as $r^{3-\gamma+\alpha}$ instead of $r^{2-\gamma}$ (that is the resulting density function after randomly shifting the first points). It follows immediately that $f^{*}(r)$ behaves as $r^{3-\gamma+\alpha+(\alpha+1)}=r^{4-\gamma+2 \alpha}$. This corresponds to a singularity of $\xi(r)$ of order of $\gamma-2(\alpha+1)$.

Thus, power-law distributed random shifts with density $d^{*}(r) \sim r^{\alpha}(-1<\alpha<0)$ will reduce the order of the pole of the correlation function by $2(\alpha+1)$.

\subsection{Simulating shifted point processes}

The Neyman-Scott processes are too simple to describe the observed galaxy distribution. As we have not found analytic expression for the probability density functions for more realistic point processes, we have studied the effect of random shifts on such processes by means of simulations.

The first model to analyze is the Cox segment process introduced in Sect. 2. The realization of the model has been simulated in a box of side $L=100$ with parameters $\lambda_{\mathrm{s}}=10^{-3}, \lambda_{\ell}=0.6$, and $\ell=10$ (see Fig. 2).

The second model is based on Voronoi tessellations. Van de Weygaert (1991), Van de Weygaert \& Icke (1989), Icke \& Van de Weygaert (1987) suggested that clusters of galaxies, the peaks of the matter density field, are situated at the vertices of the tessellation. It is remarkable how well this model describes the cluster distribution. When nothing else is known, one can assume a completely random distribution of the initial points, the nuclei that will
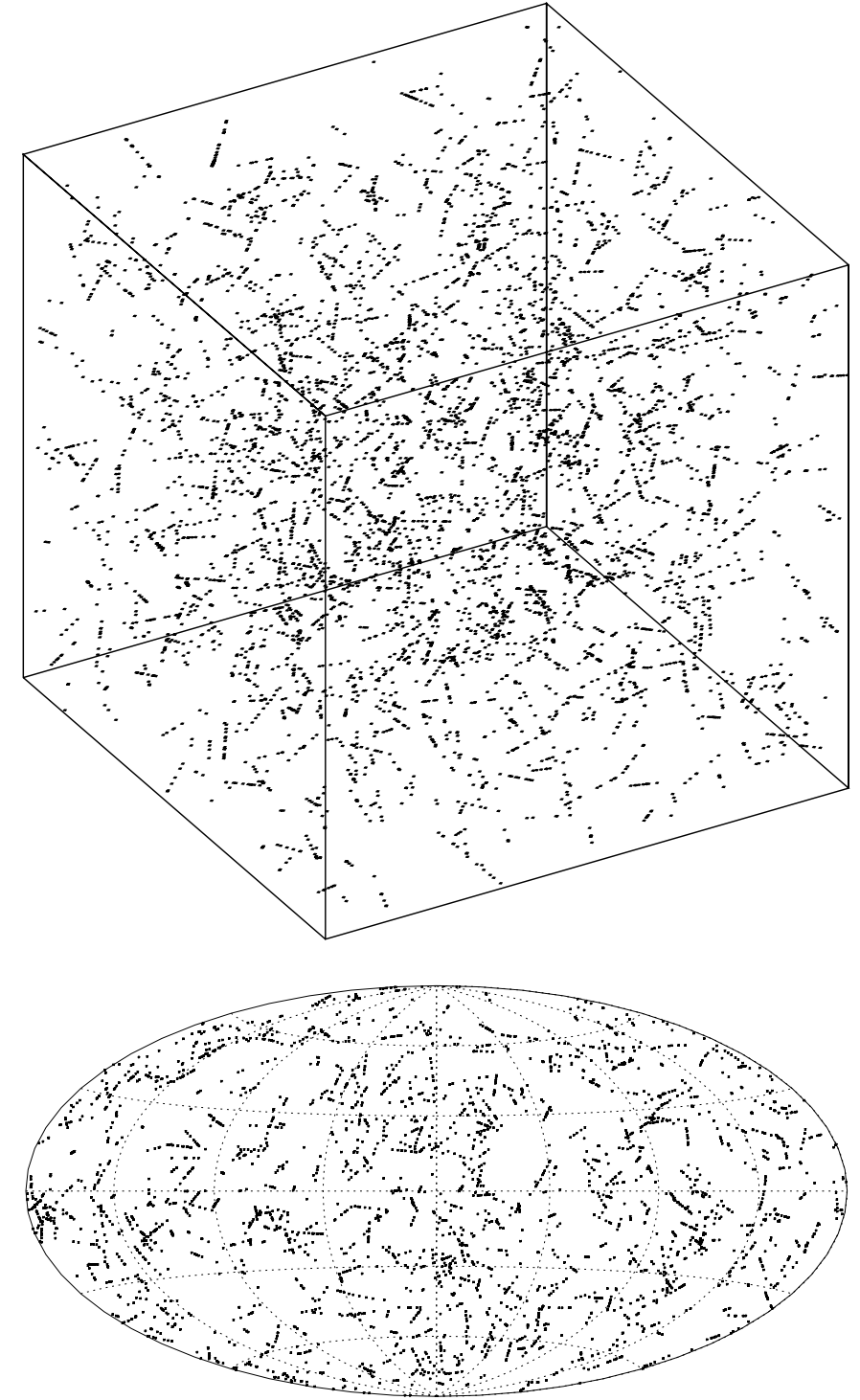

Fig. 2. The upper panel shows a realization of the segment Cox process. The bottom panel shows the Hammer-Aitoff projection of the points lying within the maximal sphere included in the cube.

become the centers of the Voronoi cells, i.e., the empty regions. Since the initial points form a homogeneous Poisson process, the tessellation is usually referred as a PoissonVoronoi tessellation. Figure 3 shows a realization of this process consisting of 10085 vertices resulting from the Voronoi tessellation of 1500 Poissonian distributed nuclei within a box of side 100 .

The determination of the two-point correlation function of the corresponding vertices process is very complicated. Heinrich et al. (1998) have shown that the singularity of $\xi(r)$ at $r=0$ is of order 2. As studies of the real galaxy point distribution suggest an order between 1.5 and 1.9 , the vertices process cannot strictly be an appropriate model for the galaxy distribution.

A quite natural way to compensate for this difference between the model and reality is to add perturbations to each point of the Voronoi galaxy model, as we did for the 

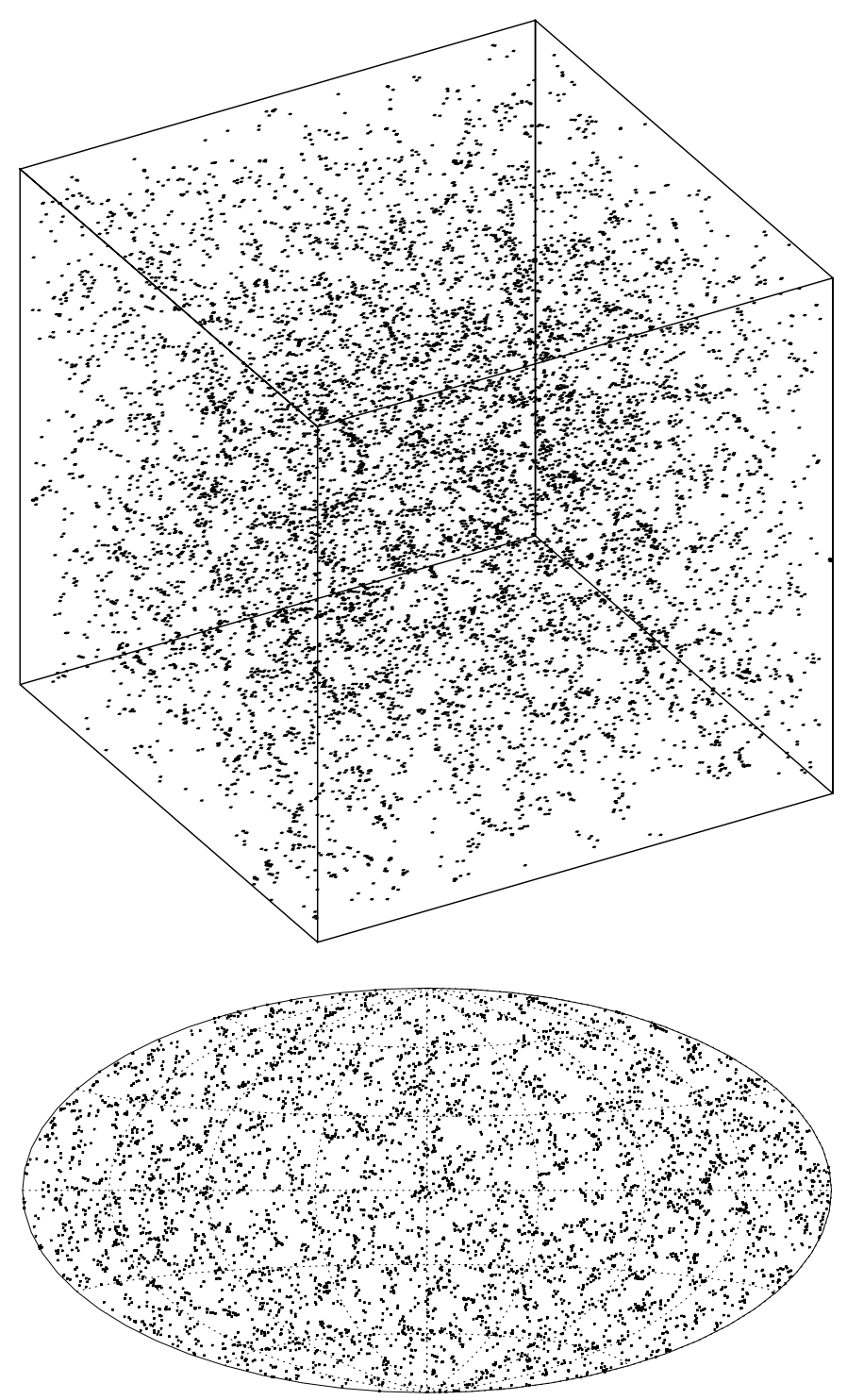

Fig. 3. The upper panel shows a realization of the a Poisson-Voronoi vertices process. The bottom panel shows the Hammer-Aitoff projection of the points lying within the maximal sphere included in the cube.

Neyman-Scott models in the previous section. That means that, after realizing a sample of this model, each point is independently and randomly shifted. This has the additional effect that the close attraction between the points is relaxed. As it seems to be plausible that the degree of the attraction between the points corresponds to the order of the singularity, the relaxation should lead to a reduction of this order.

Certainly, there is no prior theoretical distribution for the random shifts. But the results of the previous section suggest that a power-law distribution of shifts should work well. There we determined the effect of arbitrary random shifts on the order of the singularity of $\xi(r)$ in case of Neyman-Scott processes. As these processes are based on a homogeneous Poisson process as well as both the Cox segment processes and the vertices process of a PoissonVoronoi tessellation, it is plausible to assume that in all
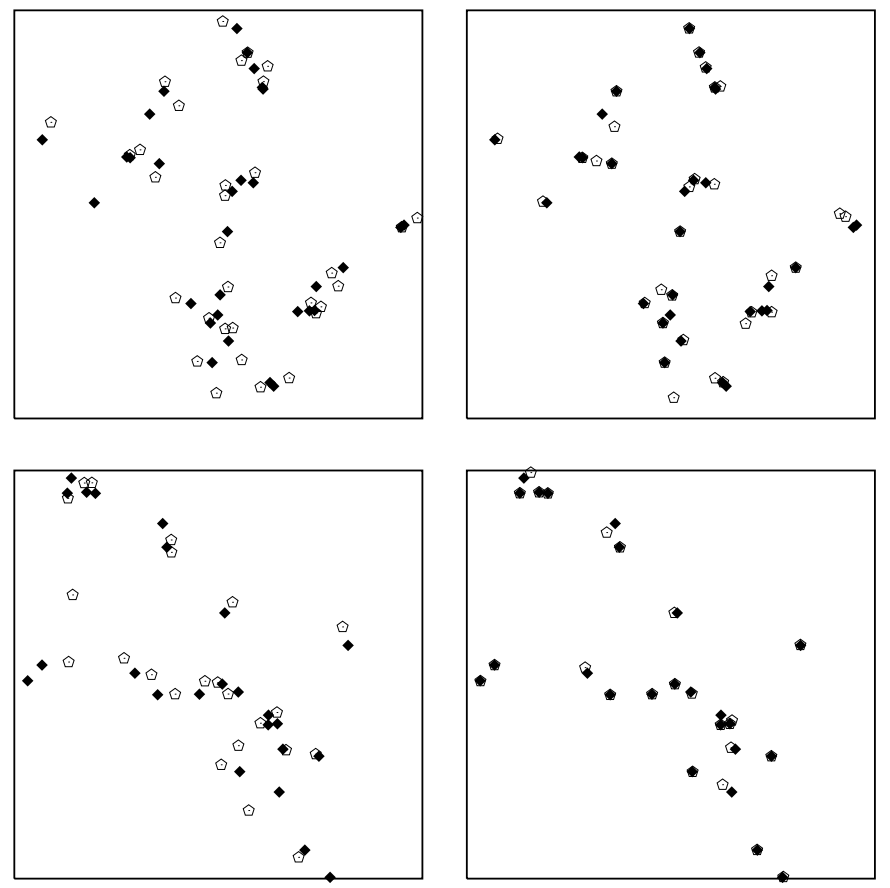

Fig. 4. Shifting Cox and Voronoi processes. The upper panels show the orthogonal projection on the bottom of a small parallelepiped, with dimensions $15 \times 15 \times 20$, drawn from the Cox segment process (Fig. 2), the lower panels show the projection on the bottom of a parallelepiped with dimensions $15 \times 15 \times 10$, drawn from the Voronoi vertices process (Fig. 3). The left panels demonstrate Gaussian shifting, the right panels demonstrate power-law shifting. The solid symbols represent the original process, the open symbols show the shifted points.

cases the effect of random shifts is similar. Thus, also in case of the simulated processes, any suitable density function $d^{*}(r)$ should lead to a reduction of the order of the singularity.

We study this conjecture by simulation. We started with the simulated Cox segment process and the PoissonVoronoi vertices process and applied random shifts. The random shifts used are distributed according to the probability density function

$d^{*}(r)= \begin{cases}0.25 r^{-0.75} & \text { for } 0 \leq r \leq 1 \\ 0 & \text { otherwise }\end{cases}$

The effects of the power-law shifts are demonstrated in Fig. 4 (right panels).

For comparison, we also tried shifting the points by Gaussian-distribution shifts. As Gaussian distribution is commonly used when modelling an unknown random effect, we had hoped that it can serve as a useful reference model. Thus, given the simulated point fields, both the Cox segment process and the Poisson-Voronoi vertices process, every point is independently shifted by a threedimensional Gaussian distributed vector with $\sigma=0.5$. Figure 4 (left panels) shows the result of this procedure.

Correlation functions amplitudes usually demand averaging over a rather large number of pair distances and are not easy to use near the pole. The expected power-law 
behaviour can be analyzed better using the correlation integral - the so-called $K$-function,

$K(r)=\int_{0}^{r} 4 \pi s^{2}[1+\xi(s)] \mathrm{d} s$.

If $K(r)$ behaves as a power law, we can define the correlation dimension $D_{2}$ as the exponent of the relation $K(r) \propto r^{D_{2}}$.

We used Ripley's estimator for a cuboidal window (Baddeley et al. 1993):

$\hat{K}(r)=\frac{V}{N^{2}} \sum_{i=1}^{N} \sum_{\substack{j=1 \\ j \neq i}}^{N} \frac{\theta\left(r-\left|\boldsymbol{x}_{i}-\boldsymbol{x}_{j}\right|\right)}{\omega_{i j}}$,

where $V$ is the volume of the cube, $N$ is the total number of points, $\theta(\cdot)$ is the Heaviside's step function and the weights $\omega_{i j}$ account for the edge correction, being the conditional probability that point $j$ would be observed given that it lies at distance $r$ from point $i$.

If we find all the distances $r_{i j}=\left|\boldsymbol{x}_{i}-\boldsymbol{x}_{j}\right|$ and the corresponding weights $\omega_{i j}$, we can order them by increasing distance, and define the ordered set $\left\{r_{\alpha}\right\}_{\alpha=1}^{N^{2}}$ with the attached weights $\left\{\omega_{\alpha}\right\}_{\alpha=1}^{N^{2}}$, then the estimator (9) can be rewritten as

$\hat{K}\left(r_{\alpha} \leq r<r_{\alpha+1}\right)=\frac{V}{N^{2}} \sum_{\beta=1}^{\alpha} \frac{1}{\omega\left(r_{\beta}\right)}$,

for even indices $\alpha$, since each $r_{\alpha}$ value is repeated twice. This is the sample estimate of the cumulative probability distribution function of pairwise separations. For a large point sample the function (10) demands a huge memory storage. In practice we can approximate this function by logarithmic discretization of the total pairwise distance interval.

The results are shown in Fig. 5. The $K$-function is shown as a staircase, and the straight lines correspond to the expected correlation dimensions $\left(D_{2}=1\right.$ for pure samples, $D_{2}=3-\gamma+2(1+\alpha)=1.5$ for shifted samples). We can clearly see that $D_{2}$ is extremely close to the expected value. The small differences between the expected power law and the real $K(r)$ dependence are probably due to the finiteness of the simulated samples and the algorithmic (precision) problems in modelling singular point distributions. The correlation integral for the Gaussianshifted samples does not reach near the pole and has the (Poisson) correlation dimension $D_{2} \approx 3$, indicating that the correlation structure has been destroyed.

We have also calculated directly the pair correlation functions: these do not reach to the smallest pair distances, as explained above. Figure 6 shows the pair correlation functions $g(r)=\xi(r)+1$ for the Cox process and the Voronoi vertices process, both before and after the random shifts. We have tried different estimators (see Pons-Bordería et al. 1999), but no significant differences were found amongst them. The correlation functions given in Fig. 6 were calculated using the Rivolo estimator
(Rivolo 1986). As in the previous figure, the straight lines correspond to the expected order of singularity $(\gamma=2$ for pure samples, $\gamma=1.5$ for shifted samples). We can see that $\gamma$ lies in the expected range, supporting the conjecture that the reduction of the order of the singularity by $2(1+\alpha)$, using random shifts with the power-law density $d^{*}(r) \sim d^{\alpha}$ is a universal phenomenon.

We also see that Gaussian shifts have completely destroyed the singularity.

\section{Summary and conclusions}

1. This paper is based on the empirical evidence that the two-point correlation function $\xi(r)$ of the galaxy point field follows for small $r$ a power-law $\xi(r) \propto r^{-\gamma}$ with $1.5<\gamma<1.9$. Nevertheless, physically motivated published models for the galaxy point pattern do not have this property.

2. A reason for this dilemma may be that these models are usually based on some simplifying assumptions. As these ideal conditions are only an approximation of reality, it is not surprising that also the point field model is an approximation.

3. One way to cover the difference between theory and reality is to add a further random element to the theoretical point field model, i. e. to randomly shift every point.

4. The determination of the effect of randomly shifting on the order of the singularity of $\xi(r)$ is too complicated for the general case. Therefore, this paper has investigated this question only for a relatively simple class of Poisson cluster processes, the Neyman-Scott processes. In this case, there is an easy rule how to choose the distribution of the random shifts to get any desired reduction of the order of the singularity. If $d^{*}(r)$, the distribution of the length of the isotropic shifts, behaves for small $r$ as $r^{\alpha}$, then the order is reduced by $2(1+\alpha)$. Unfortunately, Neyman-Scott processes are not variable enough to model the galaxy point pattern.

5. The vertices process of a Poisson-Voronoi tessellation and the Cox segment process represent the galaxy clustering better, but their two-point correlation functions have singularities of order $\gamma=2$. This is too large. Simulation studies support the conjecture that randomly shifting the vertices by power-law distributed random shifts leads to a reduction of $\gamma$ in the same way as it does in the case of Neyman-Scott processes.

6. Thus, a suitably shifted vertices process of a PoissonVoronoi tessellation or of the Cox segment process may be an appropriate model for the galaxy point pattern, as agrees with observations.

7. The method of randomly shifting may be applicable also in case of other initial point fields, apart from the processes studied here. This may lead to a number of reasonable models for the galaxy point pattern.

Acknowledgements. We thank the referee, Stephane Colombi, for his constructive criticism and useful suggestions. 

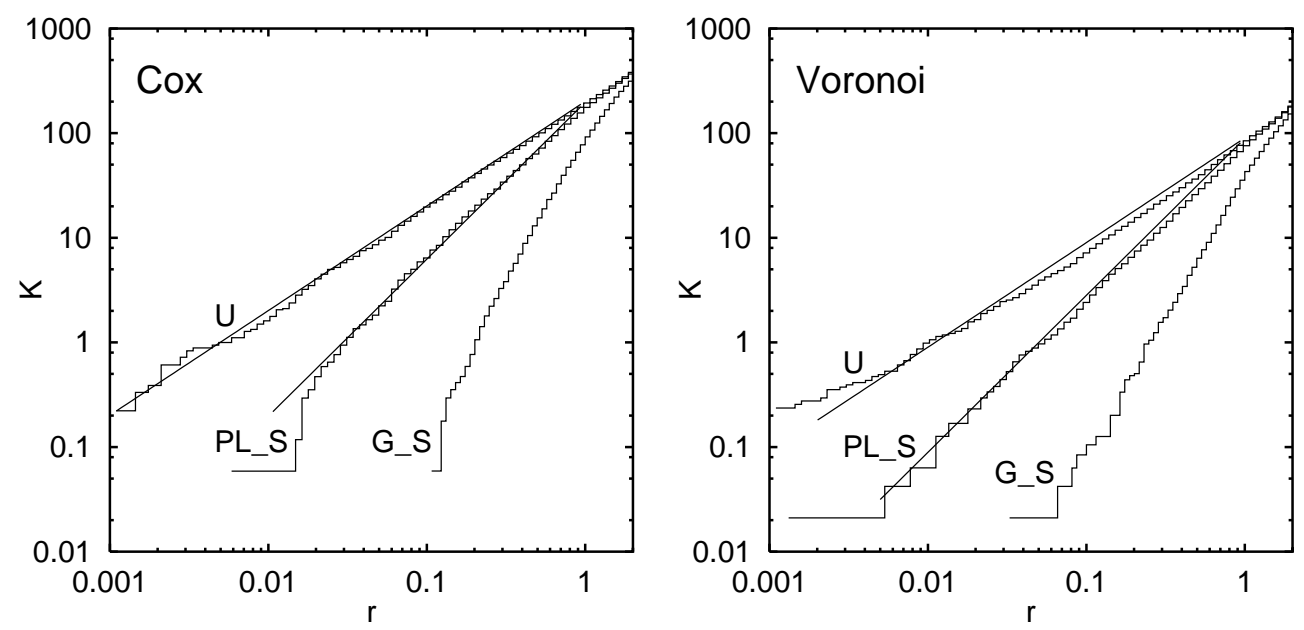

Fig. 5. Effect of shifting on the correlation integral $K(r)$. Label U stands for the unshifted original processes, label PL_S stands for the power-law shifted processes, and label G_S stands for the Gaussian shifted processes. The left panel shows the Cox family of processes, the right panel shows the Voronoi family. The straight lines represent the expected power-law behaviour.
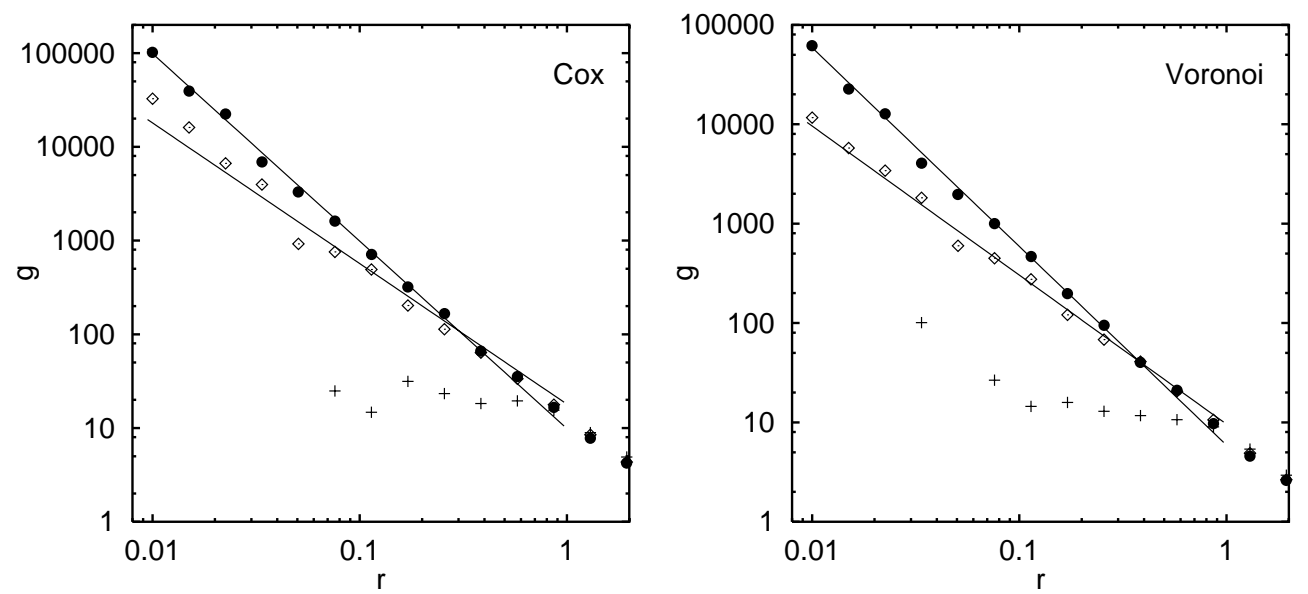

Fig. 6. Effect of shifting on the pair correlation function $g(r)$. The left panel shows the Cox family of processes, the right panel shows the Voronoi family. The correlation functions for the original processes are shown by solid bullets, for the power-law shifted processes by open diamonds, and for Gaussian-shifted processes by crosses. The straight lines represent the expected power-law behaviour.

This work was supported by the Spanish MCyT project AYA2000-2045. ES acknowledges support from the Vicerrectorado de Investigación de la Universitat de València.

\section{Appendix A: Derivation of Eq. (5)}

Here, the asymptotic behaviour of $f^{*}(r)$ for $r \rightarrow 0$ is determined. The starting point is the integral (5)

$F^{*}(r)=\int_{-\infty}^{\infty} \int_{-\infty}^{\infty} f(x) d^{*}(z) \frac{A(x ; z ; r)}{4 \pi z^{2}} \mathrm{~d} x \mathrm{~d} z$

where $f(r)$ behaves as $r^{2-\gamma}$ (i. e. the order of the singularity of $g(r)$ before the random shifts is $\gamma)$ and $d^{*}(r)$ behaves as $r^{\alpha}, \gamma<3$ and $\alpha>-1$.

Elementary considerations show that the asymptotic behaviour of $F^{*}(r)$ is equivalent to the case where $f(r)=r^{2-\gamma} \mathbb{1}_{[0,1]}(r)$ and $d^{*}(r)=r^{\alpha} \mathbb{1}_{[0,1]}(r)$, where $\mathbb{1}_{[a, b]}(r)$ is the indicator function of the interval $[a, b]$,

$$
\mathbb{1}_{[a, b]}(r)= \begin{cases}1 & \text { for } r \in[a, b] \\ 0 & \text { for } r \notin[a, b]\end{cases}
$$

Thus, the calculations are carried out only for this case.

We can write

$$
\begin{aligned}
F^{*}(r)= & \int_{0}^{r} \int_{r-z}^{r+z} \frac{r^{2}-(x-z)^{2}}{4 x^{\gamma-1} z^{1-\alpha}} \mathrm{d} x \mathrm{~d} z \\
& +\int_{r}^{1} \int_{z-r}^{z+r} \frac{r^{2}-(x-z)^{2}}{4 x^{\gamma-1} z^{1-\alpha}} \mathrm{d} x \mathrm{~d} z \\
& +\int_{0}^{r} \int_{0}^{r-z} x^{2-\gamma} z^{\alpha} \mathrm{d} x \mathrm{~d} z,
\end{aligned}
$$


and its derivative

$$
\begin{aligned}
& f^{*}(r)=\int_{0}^{r} \int_{r-z}^{r+z} \frac{r}{2 x^{\gamma-1} z^{1-\alpha}} \mathrm{d} x \mathrm{~d} z+\frac{r^{3-\gamma+\alpha}}{(3-\gamma)(4-\gamma)} \\
& -\frac{\Gamma(1+\alpha) \Gamma(3-\gamma)}{\Gamma(4+\alpha-\gamma)} r^{3-\gamma+\alpha} \\
& +\int_{r}^{1} \int_{z-r}^{z+r} \frac{r}{2 x^{\gamma-1} z^{1-\alpha}} \mathrm{d} x \mathrm{~d} z-\frac{r^{3-\gamma+\alpha}}{2^{\gamma-2}(3-\gamma)} \\
& +\frac{\Gamma(1+\alpha) \Gamma(3-\gamma)}{\Gamma(4+\alpha-\gamma)} r^{3-\gamma+\alpha} \\
& =\frac{r}{2(2-\gamma)} \int_{0}^{r} \frac{(r+z)^{2-\gamma}-(r-z)^{2-\gamma}}{z^{1-\alpha}} \mathrm{d} z \\
& +\frac{r}{2(2-\gamma)} \int_{r}^{1} \frac{(z+r)^{2-\gamma}-(z-r)^{2-\gamma}}{z^{1-\alpha}} \mathrm{d} z \\
& +\frac{r^{3-\gamma+\alpha}}{3-\gamma}\left(\frac{1}{4-\gamma}-2^{2-\gamma}\right) \\
& =\frac{r}{2(2-\gamma)} \int_{0}^{1} \frac{(r+z)^{2-\gamma}}{z^{1-\alpha}} \mathrm{d} z \\
& -\frac{r}{2(2-\gamma)} \int_{0}^{r} \frac{(r-z)^{2-\gamma}}{z^{1-\alpha}} \mathrm{d} z \\
& -\frac{r}{2(2-\gamma)} \int_{r}^{1} \frac{(z-r)^{2-\gamma}}{z^{1-\alpha}} \mathrm{d} z \\
& +\frac{r^{3-\gamma+\alpha}}{3-\gamma}\left(\frac{1}{4-\gamma}-2^{2-\gamma}\right) \\
& =\frac{r}{2(2-\gamma)}{ }_{2} F_{1}(\gamma-2, \alpha, 1+\alpha,-1 / r) r^{2-\gamma} / \alpha \\
& -\frac{r}{2(2-\gamma)} \frac{\Gamma(3-\gamma) \Gamma(\alpha)}{\Gamma(3-\gamma+\alpha)} r^{2-\gamma+\alpha} \\
& -\frac{r}{2(2-\gamma)} \frac{(1-r)^{3-\gamma}}{3-\gamma} \\
& \times{ }_{2} F_{1}(3-\gamma, 1-\alpha, 4-\gamma, 1-1 / r) r^{-1+\alpha} \\
& +\frac{r^{3-\gamma+\alpha}}{3-\gamma}\left(\frac{1}{4-\gamma}-2^{2-\gamma}\right)
\end{aligned}
$$

where ${ }_{2} F_{1}$ is the Gaussian hypergeometrical function, see Kratzer \& Franz (1960).

For small $r$ it behaves as

$$
\begin{aligned}
& { }_{2} F_{1}(\gamma-2, \alpha, 1+\alpha,-1 / r) \sim \\
& \begin{cases}\frac{\Gamma(1+\alpha) \Gamma(\gamma-2-\alpha)}{\Gamma(\gamma-2)} r^{\alpha}, & \text { if } 2-\gamma+\alpha<0, \\
\frac{\alpha}{2-\gamma+\alpha} r^{\gamma-2}, & \text { if } 2-\gamma+\alpha \geq 0,\end{cases}
\end{aligned}
$$

and

$$
\begin{aligned}
& { }_{2} F_{1}(3-\gamma, 1-\alpha, 4-\gamma, 1-1 / r) \sim \\
& \left\{\begin{array}{l}
\frac{\Gamma(4-\gamma) \Gamma(\gamma-2-\alpha)}{\Gamma(1-\alpha)(1-r)^{3-\gamma}} r^{3-\gamma}, \text { if } 2-\gamma+\alpha<0, \\
\frac{3-\gamma}{(2-\gamma+\alpha)(1-r)^{1-\alpha}} r^{1-\alpha}, \text { if } 2-\gamma+\alpha \geq 0 .
\end{array}\right.
\end{aligned}
$$

When $2-\gamma+\alpha<0$, all terms behave as $r^{3-\gamma+\alpha}$. When $2-\gamma+\alpha \geq 0$, all linear terms cancel each other out. Thus, in any case there remain only terms which behave as $r^{3-\gamma+\alpha}$ and so does $f^{*}(r)$.

\section{References}

Baddeley, A. J., Moyeed, R. A., Howard, C. V., \& Boyde, A. S. 1993, Appl. Statist., 42, 641

Bhavsar, S. P., \& Ling, E. N. 1988, ApJ, 331, L63

Buryak, O., \& Doroshkevich, A. 1996, A\&A, 306, 1

Davis, M., \& Peebles, P. J. E. 1983, ApJ, 267, 465

Heinrich, L., Körner, R., Mehlhorn, N., \& Muche, L. 1998, Statistics, 31, 235

Icke, V., \& Van de Weygaert, R. 1987, A\&A, 184, 16

Kauffmann, G., Colberg, J. M., Diaferio, A., \& White, S. D. M. 1999, MNRAS, 303, 188

Kerscher, M. 2001, Phys. Rev. E, 64, 056109

Kerscher, M., Mecke, K., Schuecker, P., et al. 2001, A\&A, 377, 1

Kratzer, A., \& Franz, W. 1960, Transzendente Funktionen (Leipzig: Akademische Verlagsgesellschaft Geest \& Portig K.-G.)

Maddox, S. J., Efstathiou, G., Sutherland, W. J., \& Loveday, J. 1990, MNRAS, 242, 43P

Mandelbrot, B. B. 1982, The Fractal Geometry of Nature (San Francisco: W.H. Freeman)

Martínez, V. J., Portilla, M., Jones, B. J. T., \& Paredes, S. 1993, A\&A, 280, 5

Martínez, V. J., \& Saar, E. 2002, Statistics of the Galaxy Distribution (Boca Raton: Chapman and Hall/CRC press)

Neyman, J., \& Scott, E. L. 1958, J. Roy. Statist. Soc. B, 20, 1

Peebles, P. J. E. 1980, The Large Scale Structure of the Universe (Princeton: Princeton Univ. Press)

Pons-Bordería, M.-J., Martínez, V. J., Stoyan, D., Stoyan, H., \& Saar, E. 1999, ApJ, 523, 480

Rivolo, A. R. 1986, ApJ, 301, 70

Scoccimarro, R., \& Sheth, R. K. 2002, MNRAS, 329, 629

Soneira, R. M., \& Peebles, P. J. E. 1978, AJ, 83, 845

Stoyan, D. 1992, Metrika, 39, 67

Stoyan, D. 1994, Statistics, 25, 267

Stoyan, D., Kendall, W., \& Mecke, J. 1995, Stochastic Geometry and its Applications (Chichester: J. Wiley \& Sons)

Szapudi, I., \& Colombi, S. 1996, ApJ, 470, 131

Van de Weygaert, R. 1991, MNRAS, 249, 159

Van de Weygaert, R., \& Icke, V. 1989, A\&A, 213, 1 